Research Journal of Biological Sciences 5 (3): 241-245, 2010

ISSN: $1815-8846$

(C) Medwell Journals, 2010

\title{
Assessment of Iranian Dental Lecturers Attitude and Perspectives Toward Objective Structured Clinical Examination (OSCE)
}

\author{
${ }^{1}$ Elaheh Vahid Dastjerdie, ${ }^{2} \mathrm{Abolfazl}$ Saboury, ${ }^{2}$ Mina Mahdian and ${ }^{2}$ Mohammad Javad Khahrazi Fard \\ ${ }^{1}$ Department of Orthodontics, ${ }^{2}$ Department of Prosthodontics, Dental School, \\ Shahid Beheshti University of Medical Sciences, Tehran, Iran
}

\begin{abstract}
Assessment of clinical skills has a central role in dental education. OSCE has been introduced as an effective method to provide a reliable assessment in this regard. This study aimed to describe lecturer's attitude and perceptions toward the annual OSCE implemented in an Iranian dental school. Faculty member's opinions from 10 departments of Dental School, Shahid Beheshti University of Medical Sciences in Iran were collected after the winter OSCE in 2010 using a structured questionnaire. The questionnaire consisted of 8 statements and the faculty members were asked to rate them from strongly agree to strongly disagree in Likert's scale. Data were retrieved and the frequency of responses were calculated and subjected to analysis using SPSS 16 . The response rate was $67.1 \%$. The overall attitude of the faculty members were positive toward the exam and did not show statistically significant difference between departments $(p=0.163)$. About $84.4 \%$ of the lecturers found OSCE a time consuming method nevertheless, $71.3 \%$ admitted that bias is significantly reduced in this method of evaluation. Furthermore, $94.2 \%$ of the faculty members confirmed the necessity of education and standardization of the examiner in designing a reliable OSCE. Although, OSCE is a time consuming and costly method, if designed and implemented by a group of professional and well educated staff, it is a reliable method to assess the clinical skills and capabilities of dental students.
\end{abstract}

Key words: OSCE, faculty, perception, clinical skills, assessment, Iranian dental school

\section{INTRODUCTION}

Assessment of clinical competence and communication skills with patients has always been in the center of attention among teachers and faculty members when evaluating the expected learning outcomes of medical education (Troncon, 2004; Conigliaro and Stratton, 2010). Several assessment tools have been proposed to evaluate the clinical skills of medical and dental students in the guideline released by the Association for Medical Education in Europe (AMEE) in 2003 (Shumway and Harden, 2003). Traditional formats of clinical examinations lack objectivity as the students are assessed on different patients by different examiners who mark the candidates subjectively. The Objective Structured Clinical Examination (OSCE) has been shown to be a valid and reliable assessment instrument in areas such as history taking, physical examination, treatment planning and management in a comprehensive, consistent and structured manner (Crossley et al., 2002; McLaughlin et al., 2006; Ruesseler et al., 2010). It is a multi-station performance-based examination format for objectively assessing clinical competence in a structured, standardised manner (Agarwal et al., 2010). The number and length of stations can vary depending on the format in use. However, an average OSCE is said to consist of 15-20 stations which students rotate individually (Alinier, 2003). Each station presents part of a case or problem using simulated/standardised patients, slides, audiotapes, photographs or laboratory reports and requires examinees to perform a specific procedure, solve a problem or record requested findings (Harden, 1988; Agarwal et al., 2010).

Since its introduction, OSCE has widely been applied to different levels of training i.e., undergraduate, postgraduate and national board exams in many disciplines of healthcare education and health professionals have begun to debate many aspects of this process (Rushforth, 2007). Although, it provides an objective evaluation of a wide range of clinical competencies with reduced risk of examiner bias developing a reliable OSCE requires extensive resources such as personnel and facilities, funding, support from the faculty, the administrative authority and students (Hodges et al., 2002a, b; Watson et al., 2002; Schuwirth et al., 2003; Shumway and Harden, 2003;

Corresponding Author: Abolfalz Saboury, Department of Prosthodontics, Dental School, Shahid Beheshti University of Medical Sciences, Tehran, Iran 
Bartfay et al., 2004). Dental education has undergone drastic changes over the recent years and the authorities have been incorporating innovative concepts of education and evaluation into dental curriculum and the educational programs. Since its introduction, OSCE has been regarded as a successful method of evaluation in different academic settings however, there is uncertainty regarding the validity, reliability and efficacy of the current format implemented in Iranian dental Schools. This study aims to assess the dental faculties' attitude toward OSCE in Shahid Beheshti University of Medical Sciences in 2010 .

\section{MATERIALS AND METHODS}

About 103 dental faculty members from Shahid Beheshti University of Medical Sciences, Tehran, Iran were invited to participate in this descriptive cross sectional survey. Those who were not familiar with the OSCE were excluded. So as not to influence the candidate's response, the questionnaires were anonymous.

The questionnaires were distributed and collected on the due date of winter OSCE in 2010. The OSCE took place simultaneously in 10 departments (i.e., Oral medicine, Periodontics, Oral and maxillofacial surgery, Oral radiology, Prosthodontics, Operative dentistry, Endodntics, Pedodontics, Orthodontics and Oral pathology). The format of the exam in each department was determined according to the requirements and curriculum materials of the specific program. The questionnaire was developed based on a comprehensive literature review and modified from a previously validated instrument used to evaluate a similar group of students (Noohi and Motesaddi, 2009).

To ensure content and construct validity, two senior faculty members with experience in dental education, curriculum design and evaluation, reviewed the instrument. Modifications were made to better fit the study objectives and ambiguous items were clarified. The instrument then was reviewed again and further refined for use in the study. It consisted of eight close ended questions and the faculty members were asked to rate each question in a 5 -score Likert scale from strongly agree to strongly disagree. The relative frequency of responses in each department was calculated and subjected to Kruskal Wallis test to determine statistical significance between the groups.

\section{RESULTS AND DISCUSSION}

The response rate among faculty members was $67.1 \%$ $(\mathrm{N}=68)$. Table 1 shows the frequency (\%) of responses to each question. About $37.9 \%$ of faculty members found the OSCE an effective tool to assess communication skills and patient management. A considerable proportion of the examiners $(84.4 \%)$ believed that OSCE is a time consuming method of evaluation yet $71.3 \%$ confirmed that bias is significantly reduced in OSCE compared to other means of evaluation. The majority of faculty members (92.4\%) confirmed the necessity of standardization among the faculty members for designing and conducting a reliable OSCE.

The overall attitude of the lecturers toward OSCE was positive and did not show any significant difference between different departments ( $p=0.163$ ). OSCEs have a central role in providing a formative evaluation of clinical competence and patient management skills plays in different clinical disciplines including dentistry (Hodges et al., 2002a; Mavis and Henry, 2002). In order to develop and expand the use of OSCE, it is necessary to identify the associated limitations and weaknesses of its application (Hodges et al., 2002b). Thus, researcher sought to evaluate dental facultie's perception and attitude toward this method in Shahid Beheshti University of Medical Sciences.

Troncon (2004) proposed that absence of a favorable attitude toward innovative educational programs among students and faculty members in Brazil may ascribe for the limitations of implementing OSCE for clinical evaluation. However, the findings demonstrated a positive attitude among the faculty members of Shahid Beheshti School of Dentistry in Iran which was consistent with that of Noohi's in Kerman university of Medical Sciences, Kerman, Iran (Noohi and Motesaddi, 2009). This indeed describes the positive trend toward educational development strategies among Iranian dental schools. Other studies have further

Table 1: OSCE questions and frequency of responses

\begin{tabular}{|c|c|c|c|c|c|}
\hline Questions & Strongly agree & Agree & Neutral & Disagree & Strongly disagree \\
\hline OSCE is an effective tool to evaluate different aspects of clinical skills & 19.7 & 51.5 & 16.7 & 12.1 & 0.0 \\
\hline OSCE is an effective tool to assess communication skills and patient managment & 7.6 & 30.3 & 30.3 & 24.2 & 7.6 \\
\hline OSCE provides an objective evaluation of the student's clinical competencies & 16.7 & 47.0 & 18.2 & 15.2 & 3.0 \\
\hline OSCE reduces bias in clinical evaluation & 16.7 & 57.6 & 22.7 & 1.5 & 1.5 \\
\hline OSCE is more time consuming compared to other methods & 40.9 & 43.9 & 7.6 & 4.5 & 3.0 \\
\hline The more the stations, the higher the validity of $\mathrm{OSCE}$ & 25.8 & 45.5 & 12.1 & 16.7 & 0.0 \\
\hline The less the stations, the higher the specificity and clarity of answers & 7.6 & 16.7 & 33.3 & 33.3 & 9.1 \\
\hline OSCE requires specific training and standardization of the faculty members & 54.5 & 37.9 & 6.1 & 1.5 & 0.0 \\
\hline
\end{tabular}


confirmed the success of OSCE among other assessment methods (Fields et al., 2007). Regarding the efficacy of OSCE as a reliable means of evaluating communication and patient management skills, the majority of faculty members stated that in the presence of a Standardized Patient (SP), this aspect of clinical competence would effectively be assessed by OSCE. SPs could be lay persons trained to portray a scripted patient presentation in a standardized and consistent fashion or a healthcare professional i.e., senior residents or faculty members (Wang et al., 2004; Iramaneerat et al., 2008). In the modified OSCE carried out in 2009 in Pakistan, inclusion of standard patient as one of the items of the exam was shown to be effective in evaluating different aspects of clinical competence including patient management (Iqbal et al., 2009). Cannick et al. (2007) performed an OSCE to evaluate the communication skills and tobacco cessation counseling technique of dental students and reported that OSCE is a unique evaluation tool that can be used to provide a standardized assessment of student's competency in several areas of dental education including communication skills, medical history taking and treatment planning (Cannick et al., 2007). Other studies have also shown the use of standardized patients to be effective in evaluating patient management skills in pediatric dentistry and geriatric practice (Fabiny et al., 1998; Zartman et al., 2002; Tung and Thomas, 2009). Although, training proctors (dentist or non-dentist) to act as a standardized patient may be associated with specific costs, the benefits accrued can easily repay the efforts and resource expenditure (Kay et al., 1994; Wallace et al., 2002; Amano et al., 2004).

In the present study, most faculty members believed that OSCE is a less-prone-to-bias method of evaluating clinical competencies compared to other evaluation tools (Table 1). Developing a standard check list for the examiners/raters that includes all the tasks the student is expected to demonstrate, renders a fair scoring process which enables the faculty members to judge the student's clinical knowledge or performance (Doig et al., 2000; Zartman et al., 2002).

Numerous studies have documented the fact examination bias is significantly reduced in a well designed OSCE (El-Nemer and Kandeel, 2009; Iqbal et al., 2009). However, some researchers believe that OSCEs are vulnerable to systematic biases due to rater fatigue. Incorporating frequent rest periods for the examiners and involving multiple raters to evaluate the examinee's performance based on a standard checklist could reduce the possibility of such errors (Humphris and Kaney, 2001; McLaughlin et al., 2009). Regarding the number of stations, researcher found limited data to dispute whether it has any effects on the quality of OSCE. Evidence suggests that the number of stations should be determined by the curricular material and the time available to perform the OSCE (Zartman et al., 2002; Faryabi and Farzad, 2010). In the present study, however the majority of researchers believed that more stations increases the internal validity of the OSCE (Table 1). OSCE in dentistry may include written or practical stations. Thus, a professional team of specialists in each specific program of dentistry and experts in dental education should be responsible for designing and implementing the OSCE and training the standard patients. The majority of the faculty members in this study unanimously believed that designing a robust, reliable and standard OSCE requires more time and specific trainings and standardization of the organizing team.

This finding was consistent with other reports which documented the high costs associated with implementing a successful OSCE (Holmboe and Hawkins, 1998; Barman, 2005; Humani and Foroughi, 2008; Iqbal et al., 2009). However, despite all the extensive resources and the expenditure, OSCE has been shown to be the most reliable means of evaluating practical and clinical skills in different disciplines of healthcare education and thus should be further developed to meet all the requirements of a successful assessment tool (Furlong et al., 2005; Saboury and Dastjerdie, 2010).

\section{CONCLUSION}

The results indicate that although, the faculty members found OSCE an expensive and time consuming method if designed and implemented by a professional team, it can render valuable results in evaluating student's clinical knowledge and performance.

\section{REFERENCES}

Agarwal, A., B. Batra, A.K. Sood, R. Ramakantan and S.K. Bhargava et al., 2010. Objective structured clinical examination in radiology. Indian J. Radiol. Imaging, 20: $83-88$.

Alinier, G., 2003. Nursing students and lecturers perspectives of objective structured clinical examination incorporating simulation. Nurse Educ. Today, 23: 419-426.

Amano, H., T. Sano, K. Gotoh, S. Kakuta and T. Suganuma et al., 2004. Strategies for training standardized patient instructors for a competency exam. J. Dent. Educ., 68: 1104-1111.

Barman, A., 2005. Critiques on the objective structured clinical examination. Ann. Acad. Med., 34: 478-482. 
Bartfay, W.J., R. Rombough, E. Howse and R. Leblanc, 2004. Evaluation. The OSCE approach in nursing education. Can. Nurse, 100: 18-23.

Cannick, G.F., A.M. Horowitz, D.R. Garr, S.G. Reed and B.W. Neville et al., 2007. Use of the OSCE to evaluate brief communication skills training for dental students. J. Dent. Educ., 71: 1203-1209.

Conigliaro, R.L. and T.D. Stratton, 2010. Assessing the quality of clinical teaching: A preliminary study. Med. Educ., 44: 379-386.

Crossley, J., G. Humphris and B. Jolly, 2002. Assessing health professionals. Med. Educ., 36: 800-804.

Doig, C. J., P.H. Harasym, G.H. Fick and J.S. Baumber, 2000. The effects of examiner background, station organization and time of exam on OSCE scores assessing undergraduate medical students physical examination skills. Acad. Med., 75: S96-S98.

El-Nemer, A. and N. Kandeel, 2009. Using OSCE as an assessment tool for clinical skills: Nursing students feedback. Aust. J. Basic Appl. Sci., 3: 2465-2472.

Fabiny, A., P. McArdle, T. Perls, T. Inui and M. Sheehan, 1998. The geriatric objective structured clinical exercise -- A teaching tool in a geriatrics curriculum. Gerontol. Geriatr. Educ., 18: 63-70.

Faryabi, J. and M. Farzad, 2010. Dental students perspective on objective structured clinical examination in kerman university of medical sciences. J. Med. Educ. Dev. Center, 6: 34-39.

Fields, H.W., M.L. Rowland, K.W.L. Viged and S.S. Hujacd, 2007. Objective structured clinical examination use in advanced orthodontic dental education. Am. J. Orthod. Dentofacial Orthop., 131: 656-663.

Furlong, E., P. Fox, M. Lavin and R. Collins, 2005. Oncology nursing students views of a modified OSCE. Eur. J. Oncol. Nurs, 9: 351-359.

Harden, R.M., 1988. What is an OSCE? Med. Teach., 10: $19-22$.

Hodges, B., M. Hanson, N. McNaughton and G. Regehr, 2002a. Creating, monitoring and improving a psychiatry OSCE: A guide for faculty. Acad. Psychiatry, 26: 134-161.

Hodges, B., N. McNaughton, G. Regehr, R. Tiberius and M. Hanson, 2002b. The challenge of creating new OSCE measures to capture the characteristics of expertise. Med. Educ., 36: 742-748.

Holmboe, E.S. and R.E. Hawkins, 1998. Methods for evaluating the clinical competence of residents in internal medicine: A review. Ann. Intern. Med., 129: 42-48.
Humani, F. and S. Foroughi, 2008. Using OSCE method for clinical evaluation of medical students. AFRA J. Aligoudars Nurs. Fac., 3: 67-74.

Humphris, G.M. and S. Kaney, 2001. Examiner fatigue in communication skills objective structured clinical examinations. Med. Educ., 35: 444-449.

Iqbal, M., B. Khizar and Z. Zaidi, 2009. Revising an objective structured clinical examination in a resource-limited Pakistani Medical School. Educ. Health, 22: 209-209.

Iramaneerat, C., R. Yudkowsky, C.M. Myford and S.M. Downing, 2008. Quality control of an OSCE using generalizability theory and many-faceted Rasch measurement. Adv. Health Sci. Educ. Theory Pract., 13: 479-493.

Kay, C.J., J.A. Johnson and K.C. Kopp, 1994. Standardized patients for teaching geriatric dentistry. Spec. Care Dentist., 14: 229-232.

Mavis, B.E. and R.C. Henry, 2002. Between a rock and a hard place: Finding a place for the OSCE in medical education. Med. Educ., 36: 408-409.

McLaughlin, K., L. Gregor, A. Jones and S. Coderre, 2006. Can standardized patients replace physicians as OSCE examiners? BMC Med. Educ., 6: 12-12.

McLaughlin, K., M. Ainslie, S. Coderre, B. Wright and C. Violato, 2009. The effect of differential rater function over time (DRIFT) on objective structured clinical examination ratings. Med. Educ., 43: 989-992.

Noohi, E. and M. Motesaddi, 2009. Clinical instructors perspective on objective structured clinical examination in kerman university of medical sciences. IJME, 8: 113-120.

Ruesseler, M., M. Weinlich, C. Byhahn, M.P. Muller, J. Junger, I. Marzi and F. Walcher, 2010. Increased authenticity in practical assessment using emergency case OSCE stations. Adv. Health Sci. Educ., 15: 81-95.

Rushforth, H.E., 2007. Objective structured clinical examination (OSCE): Review of literature and implications for nursing education. Nurse Educ. Today, 27: 481-490.

Saboury, A. and E.V. Dastjerdie, 2010. Dental residents attitude toward objective structured clinical examination (OSCE) as a clinical evaluation method. J. Dent. Sch., 28: 88-94.

Schuwirth, L.W.T. and C.P.M. van der Vleuten, 2003. The use of clinical simulations in assessment. Med. Educ., 37: 65-71.

Shumway, J.M. and R.M. Harden, 2003. AMEE Guide No. 25: The assessment of learning outcomes for the competent and reflective physician. Med. Teach., 25: $569-584$. 
Troncon, L., 2004. Clinical skills assessment: Limitations to the introduction of an OSCE (objective structured clinical examination) in a traditional Brazilian medical school. Sao Paulo Med. J., 122: 12-17.

Tung, E. and M. Thomas, 2009. Use of a geriatric home visit experience to teach medical students the functional status assessment. J. Gen. Inter. Med., 24: 244-246.

Wallace, J., R. Rao and R. Haslam, 2002. Simulated patients and objective structured clinical examinations: Review of their use in medical education. Adv. Psychiatr Treat, 8: 342-348.
Wang, W.D., P.C. Yang, C.Y. Chen, B.H. Lue and P.M. Yang, 2004. Using senior residents as standardized patients for evaluating basic clinical skills of medical students. J. Formos Med. Assoc., 103: 519-525.

Watson, R., A. Stimpson, A. Topping and D. Porock, 2002. Clinical competence assessment in nursing: A systematic review of the literature. J. Adv. Nurs., 39: 421-431.

Zartman, R.R., A.G. McWhorter, N.S. Seale and W.J. Boone, 2002. Using OSCE-based evaluation: Curricular impact over time. J. Dent. Educ., 66: $1323-1330$. 